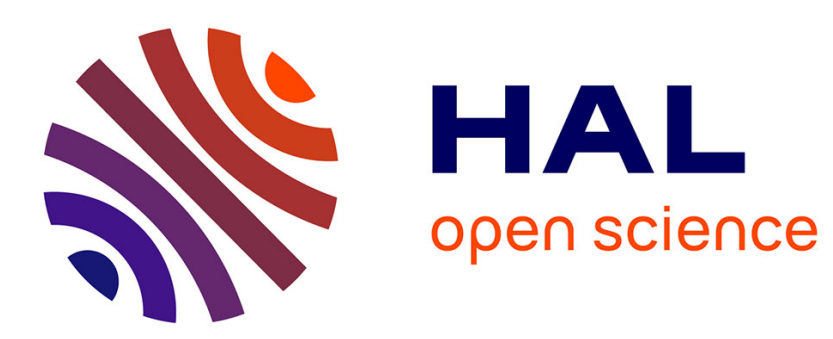

\title{
Model of Kinematic Waves for Gas-Liquid Segregation with Phase Transition in Porous Media
}

Stéphane Zaleski, Mikhail Panfilov

\section{To cite this version:}

Stéphane Zaleski, Mikhail Panfilov. Model of Kinematic Waves for Gas-Liquid Segregation with Phase Transition in Porous Media. Journal of Fluid Mechanics, 2017, 829, pp.659-680. 10.1017/jfm.2017.556 . hal-03035196

\section{HAL Id: hal-03035196 https://hal.sorbonne-universite.fr/hal-03035196}

Submitted on 2 Dec 2020

HAL is a multi-disciplinary open access archive for the deposit and dissemination of scientific research documents, whether they are published or not. The documents may come from teaching and research institutions in France or abroad, or from public or private research centers.
L'archive ouverte pluridisciplinaire HAL, est destinée au dépôt et à la diffusion de documents scientifiques de niveau recherche, publiés ou non, émanant des établissements d'enseignement et de recherche français ou étrangers, des laboratoires publics ou privés. 


\title{
Model of Kinematic Waves for Gas-Liquid Segregation with Phase Transition in Porous Media
}

\author{
Stephane Zaleski ${ }^{1}$ and Mikhail Panfilov ${ }^{1,2} \dagger$ \\ ${ }^{1}$ Institut Jean le Rond d'Alembert - CNRS / Sorbonne Universités, UMR 7190 \\ ${ }^{2}$ Institut Elie Cartan - CNRS / Université de Lorraine, UMR 7502
}

(Received $\mathrm{xx}$; revised $\mathrm{xx}$; accepted $\mathrm{xx}$ )

We consider the problem of gas-liquid flow with phase transition in a porous medium, governed by the buoyancy force. Free gas releases due to continuous pressure decrease. We take into account the gas expansion and the dissolution of chemical components in both phases controlled by the local phase equilibrium. We have developed an asymptotic model of flow for low pressure gradients in the form of a nonlinear hyperbolic system of first order with respect to the liquid saturation and the total flow velocity, which is the extended non-homogeneous Buckley-Leverett model. In two asymptotic cases determined by two different ratios between the characteristic times, this model is completely decoupled from pressure, i.e., the pressure enters in this model as a parameter determined through an independent formula.

The segregation problem with phase transition in a bounded domain is solved for two cases of boundary conditions. The saturation behaviour is described in terms of nonlinear kinematic waves, whose evolution follows a complex segregation scenario, which includes the wave reflection and formation of shocks. The macroscopic gas-liquid interfaces are described in terms of shock waves. The comparison with numerical simulations shows satisfactory results.

Key words: Porous media, wave propagation, hyperbolic equations, phase equilibrium, bubble flow, gas-liquid interface, liquid degassing, vaporizing, shock waves

\section{Introduction}

The flow of two-phase fluids with phase transition necessarily requires taking into account the internal composition of each phases. Indeed, the free gas appearance by liquid degassing means the transition of light components from liquid to gas state, similar to liquid formation by gas condensation, which means the transition of heavy components from gas to liquid state. We deal, thus, with two-phase multicomponent fluid. The mathematical model of such flow represents a system of PDE with respect to pressure $\mathcal{P}$, liquid saturation $\mathcal{S}$ (the volume fraction of liquid in porous space), and the concentrations of chemical species in liquid and gas $\mathcal{C}_{i}^{k}$ ( $i$ means liquid or gas, while $k$ is the identifier of chemical component).

The main difficulty in analyzing such a model originates from the fact that it contains different types of PDE with respect to various variables. It is expected that the saturation and concentrations are transported along the characteristic lines, whilst the pressure rather diffuses in all directions. The transport of saturation and concentrations is expected to create sharp forward fronts, while the pressure, being a continuous physical field, behaves rather smoothly without sharp variations. In other words, the behavior of the saturation and the species concentrations

$\dagger$ Email address for correspondence: michel.panfilov@dalembert.upmc.fr 
is governed rather by hyperbolic differential equations, while the pressure is determined by a parabolic operator. At the same time, the pressure determines the phase composition, the phase transitions and the phase compression-expansion, so that the saturation, the concentrations and the phase densities are strongly coupled with $\mathcal{P}$. Taking into account the qualitatively different methods of solving the diffusion and the convection equations, the idea of separating pressure and obtaining a closed model of convective transport for saturation and concentrations seems then to be fundamental.

The simplest way to do it has been suggested in the analytical theory of fluid displacement in porous media (oil displacement by water) Bedrikovetsky (1993), Entov (2000), Entov \& Zazovsky (1997), Orr (2002). The dependence of the phase densities on pressure was removed by assuming the ideal mixing (the mixture volume is the sum of volumes of the pure components). The dependence of species concentrations on pressure was removed simply by assuming the pressure to be constant, since the injection of water in an oil reservoir leads to the maintain of pressure. As the result, the species composition and phase saturation become independent of pressure. This leads to a system of hyperbolic equations for $\mathcal{S}$ and $\mathcal{C}_{i}^{k}$, which may be analyzed by the methods developed in air dynamics.

In a more advanced case, the pressure may be considered as a given linear function of coordinates Bedrikovetsky (1993), while keeping the assumption about the ideal mixing. In this case, the pressure may be separated too.

Unfortunately, in the case of phase transitions (the gas release from liquid, or the gas condensation), these assumptions are impossible to apply, as the variation of pressure in time is the main mechanism of phase transition. The ideal mixing is also inadmissible for cases of phase transitions, because the volume of a species changes significantly when it transits between liquid and gas. We have then to develop a totally different approach. It is based on the high contrast between two characteristic times of the system Panfilov (1986). The first time is that of propagation of a perturbation, $t_{p}$, while another time is that of complete fluid extraction, $t_{*}$. In real situations, time $t_{p}$ is of several days or months, while the time of complete extraction is of ten or hundred years. Then the system is characterized by a small parameter $\varepsilon=\mathrm{t}_{p} / \mathrm{t}_{*}$. Now all depends on the behaviour of the third time, which is the time of gas rising, $t_{h}$. If $t_{h} \sim t_{p}$, i.e., the fluid extraction is much slower than gas rising, then within the scale of time $t_{h}$, the pressure is weakly perturbed, so it is lowly variable in space and in time. If, in contrast, $t_{h} \sim t_{*}$, then within the scale of time $t_{h}$ the pressure is quasi-stationary. In both cases we obtain the asymptotic problems for pressure, which is independent of saturation and total velocity. Therefore, the pressure is completely separated.

The wave equations for saturation and total velocity are obtained by simple algebraic transformations of the conservations equations. They contain the pressure as a parameter, which is determined by independent formulae. The equation for the saturation represents a non-homogeneous Buckley-Leverett model (it is a classical model of two-phase immiscible flow Buckely et al. (1942)), which contains the source terms responsible for liquid degassing and gas expansion. The difference with the classical model is not only in the appearance of source terms, but in the total velocity which is non constant in our case.

Note that the first attempt to obtain the analogue of the Buckley-Leverett model for two-phase flow with oil degassing was performed in Chraibi (2008). The possibility to use the BuckleyLeverett model for systems with phase transition was also shown in Young (1993) where gas released from water in a thermal reservoir. The Buckley-Leverett equation was obtained by applying the hypothesis of small pressure gradients. However, the pressure was not decoupled.

Note that a non-homogeneous extension of the Buckley-Leverett model has been put forward in Kalisch (Mitrovic and Nordbotten).

The wave model obtained in the present paper was used to analyse the gas release from liquid and heir segregation in natural geological porous reservoirs. Beyond the well-known case of 
natural gas in oil reservoirs, this also corresponds to the air liberation from water in a thermal aquifer, or to hydrogen release from water in a natural hydrogen reservoir (Panfilov (Zaleski \& Josserand)), which are less traditional objects but represent high interest within the framework of the energy transition. At the initial state the fluid is liquid (oil or water) and contains methane or nitrogen, or hydrogen (the light component) in dissolved form. Oil or water are extracted by the system of wells, which causes the decrease of pressure and the release of free gas from the liquid. Driven by the buoyancy force, the gas rises and creates a gas cap. The rate of segregation and the gas cap creation, as well as its geometrical structure and chemical composition are of great interest.

The structure of the paper is as follows:

- first of all the problem of two-phase two-component flow is formulated;

- the variable replacement: two phase velocities are replaced by the total velocity and the fractional flow;

- the general wave model is formulated, as well as two independent methods of calculating the pressure;

- the derivation of the model, the first step: one obtains the formal wave equations for the saturation and the total velocity, as well as the general parabolic equation for the pressure; the pressure is not decoupled, for the moment;

- the second step: the pressure is decoupled for two ratios between the characteristic times;

- the first problem of segregation is solved: the fluid is extracted through the overall thickness of the reservoir, which leads to a zero total velocity;

- the analytical results are compared with numerical simulations;

- the second problem of segregation is solved: the fluid is extracted on the reservoir top only, which leads to a non-zero and non-constant total velocity; the independent problem for pressure is solved by the method of integral relationships of Karman-Pohlhausen.

\section{Problem of gas-liquid segregation in terms of kinematic waves}

\subsection{Flow equations}

To analyse the flow of liquid with gas release we have to assume that the initial liquid contains two chemical components (at least): light (1) and heavy (2). When pressure decreases due to liquid extraction, the light component releases and creates the free gas. The gas will be assumed to consist only of the light component, i.e. the heavy component does not dissolve in gas.

For the sake of simplicity, thermal effects, capillary pressure, diffusion, and deformations of porous medium are neglected. We also accept the local phase equilibrium described by Henry's law. Gas is ideal, while liquid is incompressible (the liquid compressibility is neglected with respect to that of gas).

The mass and momentum balance equations of each chemical component are, for $i=l, g$, in the one-dimensional domain $\mathfrak{D}=\{0<x<L\}$, where $L$ is the reservoir thickness:

$$
\begin{aligned}
& \phi \partial_{t}\left(\rho_{l} \mathcal{C} \mathcal{S}+\rho_{g}(1-\mathcal{S})\right)+\nabla \cdot\left(\rho_{l} \mathcal{C} \mathbf{V}_{l}+\rho_{g} \mathbf{V}_{g}\right)=-\phi \rho_{g} q_{g}-\phi \rho_{l} \mathcal{C} q_{l} \\
& \phi \partial_{t}\left(\rho_{l}(1-\mathcal{C}) \mathcal{S}\right)+\nabla \cdot\left(\rho_{l}(1-\mathcal{C}) \mathbf{V}_{l}\right)=-\phi \rho_{l}(1-\mathcal{C}) q_{l} \\
& \mathbf{V}_{i}=-\lambda_{i}(\mathcal{S})\left(\nabla \mathcal{P}-\rho_{i} \mathbf{g}\right), \quad \lambda_{i}(\mathcal{S}) \equiv \frac{K k_{i}}{\mu_{i}} \\
& \mathcal{C}=h \mathcal{P}, \\
& \rho_{g}=\gamma \mathcal{P} \quad(\gamma=\mathrm{m} R T=\text { const }) ; \quad \rho_{l}=\text { const },
\end{aligned}
$$

where $\rho_{l}, \rho_{g}$ are the densities of liquid and gas $\left[\mathrm{kg} / \mathrm{m}^{3}\right] ; \mathcal{S}$ is the liquid saturation; $\mathcal{C}$ is the 
mass fraction of the light component in liquid; $\mathcal{P}$ is the pressure; $\mathbf{V}_{l}$ and $\mathbf{V}_{g}$ are the Darcy's velocities of liquid and gas; $h$ is the Henry's constant $\left[\mathrm{Pa}^{-1}\right] ; \mathrm{m}$ is the molecular mass of the light component $[\mathrm{kg} / \mathrm{mol}] ; \mathrm{g}$ is the gravitational acceleration, $K$ is the absolute permeability, $k_{i}$ is the relative permeability of phase $i, \mu_{i}$ is the dynamic viscosity of phase $i$ (assumed to be constant); $R$ is the universal gas constant; $q_{l}$ and $q_{g}$ are the volumetric rates of extraction of liquid and gas reported to $1 \mathrm{~m}^{3}$ of the medium [1/s]; the vertical axis $x$ is directed upwards. Functions $k_{i}(\mathcal{S}) \in[0,1]$ are monotonic continuous and have the following properties:

$$
\begin{gathered}
k_{l}=0, \quad 0 \leqslant \mathcal{S} \leqslant \mathcal{S}_{*} ; \quad k_{g}=0, \quad \mathcal{S}^{*} \leqslant \mathcal{S} \leqslant 1 ; \quad k_{l}(1)=k_{g}(0)=1, \\
0<k_{l}^{\prime}\left(\mathcal{S}_{*}\right)<1, \quad-1<k_{g}^{\prime}\left(\mathcal{S}^{*}\right)<0
\end{gathered}
$$

where $\mathcal{S}_{*}$ and $\mathcal{S}^{*}$ are the percolation thresholds for liquid and gas respectively.

The system of 6 equations (1.1) determines 6 variables: $\mathcal{P}, \mathcal{S}, \rho_{g}, \mathcal{C}, \mathbf{V}_{g}$ and $\mathbf{V}_{l}$.

To formulate the boundary conditions we should take into account the following. The diffusive fluxes of gas through the bottom coming from the Earth depth and through the reservoir top (gas leakage) may be neglected during the period of reservoir exploitation. Therefore, the reservoir bottom is impermeable for both phases. This is not the case of the reservoir top, as both liquid and gas may be produced just from the reservoir top (and not through the overall reservoir thickness). In this case the fluid extraction is better to express through the boundary condition than through a distributed sources $q_{l}$ and $q_{g}$.

At the initial state the fluid is assumed to be single-phase liquid and immobile, i.e., the initial pressure is hydrostatic.

Consequently, we obtain the following general boundary and initial conditions:

$\left.\mathcal{S}\right|_{t=0}=1 ;\left.\quad \mathcal{P}\right|_{t=0}=\mathcal{P}_{*}-\rho_{l}^{0} g x ; \quad V_{g},\left.V_{l}\right|_{x=0}=0,\left.\quad V_{l}\right|_{x=L}=V_{l *},\left.\quad V_{g}\right|_{x=L}=V_{g *}$

where $\mathcal{P}_{*}$ is the initial pressure at the reservoir bottom; $V_{l *}$ and $V_{g *}$ are the velocity of liquid and gas extraction from the reservoir top.

\subsection{Introduction of the fractional flow}

The phase velocities $\mathbf{V}_{l}$ and $\mathbf{V}_{g}$ are usually replaced by the total velocity $\mathbf{V}$ through the following relationships:

$$
\mathbf{V}_{l}=\mathcal{F} \mathbf{V}+\lambda_{g} \mathcal{F} \Delta \rho \mathbf{g}, \quad \mathbf{V}_{g}=(1-\mathcal{F}) \mathbf{V}-\lambda_{g} \mathcal{F} \Delta \rho \mathbf{g},
$$

where

$$
\mathbf{V} \equiv \mathbf{V}_{l}+\mathbf{V}_{g}, \quad \mathcal{F}(\mathcal{S}) \equiv \frac{V_{l}}{V}=\frac{\lambda_{l}}{\lambda_{l}+\lambda_{g}}=\frac{k_{l}(\mathcal{S})}{k_{l}(\mathcal{S})+k_{g}(\mathcal{S}) \mu_{l} / \mu_{g}}
$$

Indeed, if we take the sum of $\mathbf{V}_{l}$ and $\mathbf{V}_{g}$ and use Darcy's law (1.1c), then: $\mathbf{V}=-\lambda \nabla \mathcal{P}+$ $\left(\lambda_{l} \rho_{l}+\lambda_{g} \rho_{g}\right)$ g. Then:

$$
-\lambda \nabla \mathcal{P}=\mathbf{V}-\left(\lambda_{l} \rho_{l}+\lambda_{g} \rho_{g}\right) \mathbf{g}, \quad \lambda \equiv \lambda_{l}+\lambda_{g}
$$

Then from Darcy's law: $\mathbf{V}_{l}=\frac{\lambda_{l}}{\lambda}\left(\mathbf{V}-\left(\lambda_{l} \rho_{l}+\lambda_{g} \rho_{g}\right) \mathbf{g}\right)+\lambda_{l} \rho_{l} \mathbf{g}=\mathcal{F} \mathbf{V}+\mathbf{g} \frac{\lambda_{g} \lambda_{l}}{\lambda}\left(\rho_{l}-\rho_{g}\right)$, which leads to (1.4).

Function $\mathcal{F}$ is the fractional flow of liquid in the case without gravity. It has the following properties: $\mathcal{F}(\mathcal{S}) \in[0,1]$, it is a monotonic nonlinear function of saturation, and $\mathcal{F}(0) \equiv 0$, when $0 \leqslant \mathcal{S} \leqslant \mathcal{S}_{*} ; \quad \mathcal{F}(1) \equiv 1$, when $\mathcal{S}^{*} \leqslant \mathcal{S} \leqslant 1$.

The physical meaning of $\mathcal{F}(\mathcal{S})$ is the ratio between the liquid flow rate $\left(Q_{l}\right)$ and the total flow 
rate $\left(Q_{l}+Q_{g}\right)$ at zero gravity forces. Indeed, let $\mathcal{F}=\frac{Q_{l}}{Q_{l}+Q_{g}}=\frac{V_{l}}{V_{l}+V_{g}}$, then using Darcy's law (in the case of zeroth gravity), we obtain (1.5).

The initial and boundary conditions (1.3) become:

$$
\begin{aligned}
& \left.\mathcal{S}\right|_{t=0}=1 ;\left.\quad \mathcal{P}\right|_{t=0}=\mathcal{P}_{*}-\rho_{l} g x ; \\
& \left.V\right|_{x=0}=0,\left.\quad \lambda_{g} \mathcal{F} \Delta \rho g\right|_{x=0}=0 \\
& \left.V\right|_{x=L}=V_{*},\left.\quad \lambda_{g} \mathcal{F} \Delta \rho g\right|_{x=L}=V_{g *} \mathcal{F}-V_{l *}(1-\mathcal{F})
\end{aligned}
$$

where $V_{*}=V_{l *}+V_{g *}$.

\subsection{Characteristic times and scales}

The process is characterized by three times:

- the time of propagation of a pressure perturbation: $\mathrm{t}_{p} \equiv \frac{\mu_{l} L^{2} \phi}{K \mathcal{P}_{*}}$;

- the time of gas rise due to the buoyancy force: $\mathrm{t}_{h} \equiv \frac{\mu_{g} L \phi}{K \Delta \rho^{0} g}, \quad \Delta \rho^{0} \equiv \rho_{l}-\rho_{g}^{0}$;

- the time of complete fluid withdrawal: $\mathrm{t}_{*} \equiv \frac{1}{q_{l}+q_{g}}$ in the case of distributed sources, or

$\mathrm{t}_{*} \equiv \frac{L}{V_{l *}+V_{g *}}$ in the case of point sources at the reservoir top.

where $\mathcal{P}_{*}$ enters in (1.3).

The main three hypotheses of this paper, related to the introduced scales, consist of the following:

$\mathcal{H} 1$ : : the ratio between the characteristic times: $t_{p} \ll t_{*}$. Then

$$
0 \leqslant \varepsilon \equiv \frac{\mathrm{t}_{p}}{\mathrm{t}_{*}} \ll 1
$$

$\mathcal{H 2 :}$ : the initial pressure distribution along $x$ is almost constant:

$$
\frac{\rho_{l}^{0} g L}{\mathcal{P}_{*}} \sim \varepsilon
$$

This assumption is valid for thin and deep reservoirs, in which the hydrostatic pressure difference over the reservoir height $\rho_{l}^{0} g L$ is negligible with respect to the initial pressure $\mathcal{P}_{*}$.

$\mathcal{H} 3$ : : Small pressure gradients: the square of pressure gradient, $(\nabla \mathcal{P})^{2}$ and $\left(\mathbf{V}_{i} \cdot \nabla \mathcal{P}\right)$ are neglected. This hypothesis is compatible with the preceding one.

The third time $t_{h}$ is the true characteristic time of the analyzed process. It may be any. The separation of pressure from the saturation is possible in two cases:

- the slow gas ascension: $\mathrm{t}_{h} \sim \mathrm{t}_{*}$;

- the fast gas ascension: $\mathrm{t}_{h} \sim \mathrm{t}_{p}$. 


\subsection{Reduction to the asymptotic model of kinematic waves}

Using the hypotheses of the previous section, it is possible to transform system (1.1) into the following system of first-order differential equations for saturation and velocity:

$$
\left\{\begin{array}{l}
\partial_{t} \mathcal{S}+\underbrace{\frac{\mathbf{V}}{\phi} \nabla \mathcal{F}}_{\mathrm{I}}-\underbrace{\frac{1}{\phi} \nabla \cdot\left(\lambda_{g} \mathcal{F} \Delta \rho \mathbf{g}\right)}_{\mathrm{II}}=-\underbrace{\frac{\mathcal{C S}(1-\mathcal{F}+r \mathcal{F})}{(1-\mathcal{C}) \mathfrak{T}}}_{\mathrm{III}}-\underbrace{\frac{\mathcal{F}(1-\mathcal{S})}{\mathfrak{T}}}_{\mathrm{IV}}+\underbrace{q_{g} \mathcal{F}-q_{l}(1-\mathcal{F})}_{\mathrm{V}}, \\
\nabla \cdot \mathbf{V}=\frac{\phi \mathcal{C} \mathcal{S}(r-1)}{(1-\mathcal{C}) \mathfrak{T}}+\frac{\phi(1-\mathcal{S})}{\mathfrak{T}}-\phi q
\end{array}\right.
$$

where $\Delta \rho=\rho_{l}-\rho_{g}(\mathcal{P}), \quad r=\frac{\rho_{l}}{\rho_{g}(\mathcal{P})}, \quad \mathfrak{T}(\mathcal{P})=-\left(\frac{1}{\mathcal{P}} \partial_{t} \mathcal{P}\right)^{-1}, \quad q=q_{l}+q_{g}$.

The initial and boundary conditions result from (1.7):

$$
\begin{cases}\left.\mathcal{S}\right|_{t=0}=1 ; & \left.\lambda_{g} \mathcal{F} \Delta \rho g\right|_{x=0}=0,\left.\quad \lambda_{g} \mathcal{F} \Delta \rho g\right|_{x=L}=V_{g *} \mathcal{F}-V_{l *}(1-\mathcal{F}), \\ \left.V\right|_{x=0}=0, & \left.V\right|_{x=L}=V_{*}\end{cases}
$$

In this system the pressure $\mathcal{P}$ is a parameter, which is determined as the solution of the independent boundary-value problem, which is different for two different cases of $t_{h}$ mentioned in the previous section.

For the case of fast gas ascension: $\mathrm{t}_{h} \sim \mathrm{t}_{p} \ll \mathrm{t}_{*}$ :

we obtain for the function $p \equiv \mathcal{P}-\mathcal{P}_{*}+\rho_{l} g x$ :

$$
\left\{\begin{array}{l}
\lambda_{l 0} \Delta p=\omega \partial_{t} p+\phi q, \quad 0<x<L \\
\left.p\right|_{t=0}=0 \\
\left.\partial_{x} p\right|_{x=0}=0 \\
\left.\partial_{x} p\right|_{x=L}=-\frac{V_{*}}{\lambda_{l 0}}
\end{array}\right.
$$

where $\quad \omega=\frac{\phi h\left(r_{*}-1\right)}{\left(1-\mathcal{C}_{*}\right)}, \quad \lambda_{l 0}=\left.\lambda_{l}(\mathcal{S})\right|_{\mathcal{S}=1}=\frac{K}{\mu_{l}}, \quad r_{*}=\frac{\rho_{l}}{\rho_{g}\left(\mathcal{P}_{*}\right)}, \quad \mathcal{C}_{*}=h \mathcal{P}_{*}$.

For the case of fast depletion $\left(\mathrm{t}_{h} \sim \mathrm{t}_{*}\right)$, the pressure is calculated as the solution to the following Cauchy's problem for an ordinary differential equation:

$$
\frac{d \mathcal{P}}{d t}\left(\frac{h \overline{\mathcal{S}}\left(\rho_{l}-\gamma \mathcal{P}\right)}{\gamma \mathcal{P}(1-h \mathcal{P})}+\frac{1-\overline{\mathcal{S}}}{\mathcal{P}}\right)=-\left(\frac{V_{*}}{\phi L}+q\right),\left.\quad \mathcal{P}\right|_{t=0}=\mathcal{P}_{*}
$$

where $\overline{\mathcal{S}} \equiv \frac{1}{L} \int_{x=0}^{x=L} \mathcal{S}(x, t) d x$. The value $\overline{\mathcal{S}}$ should be considered as known and may be accepted in an approximate way. In particular, if we assume that $\overline{\mathcal{S}}=$ const, $\rho_{l} \gg \rho_{g}$ and $\mathcal{C} \ll 1$, then equation (1.13) has an analytical solution:

$$
\mathcal{P}=\mathcal{P}_{*} e^{-\alpha t}, \quad \alpha \equiv \frac{\frac{V_{*}}{\phi L}+q}{\left(\frac{h \overline{\mathcal{S}} \rho_{l}}{\gamma}+1-\overline{\mathcal{S}}\right)}
$$

The derivation of all these relationships is given in the next section. 
The physical meaning of various terms of equation (1.10) is as follows: term I is the saturation transport forced by the non-zero total velocity; term II is the transport by the buoyancy force, while III, IV and V are the saturation "production" due to phase transition, gas expansion, and non-proportionate liquid/gas extraction. The extraction is called proportionate, if the production rates of the phases are proportional to their fractional flow. It is seen that for a proportionate extraction, term $\mathrm{V}$ disappears.

Note that the transport terms in the left-hand side are not affected by the phase transitions, which have the impact only on the source terms. Therefore, in the case without phase transitions the model transforms into the classical Buckley-Leverett with the same convective part.

Equation (1.10) is a non-homogeneous extension of the Buckley-Leverett model known in the theory of oil recovery. The extension consists of two elements:

(i) first of all, the source terms appear in the right-hand side;

(ii) Secondly, the total velocity is non-constant (it is constant in the classical Buckley-Leverett model), and is determined, in turn, from a differential equation.

\subsection{Derivation of the model. Step I: reduction to a non-separated wave form}

The derivation of the model (1.10) - (1.12) - (1.13) is done by two steps. At the first step we obtain the first-order equations (1.10) for $\mathcal{S}$ and $\mathbf{V}$ and the following problem for pressure

$$
\begin{aligned}
& -\nabla \cdot(\lambda \nabla \mathcal{P})+\nabla \cdot\left(\lambda_{l} \rho_{l} \mathbf{g}+\lambda_{g} \rho_{g} \mathbf{g}\right)=-\left(\frac{\mathcal{S C}(r-1)}{1-\mathcal{C}}+1-\mathcal{S}\right) \frac{\phi}{\mathcal{P}} \partial_{t} \mathcal{P}-\phi q \\
& \left.\left(-\lambda \nabla \mathcal{P}+\lambda_{l} \rho_{l} \mathbf{g}+\lambda_{g} \rho_{g} \mathbf{g}\right) \cdot \mathbf{n}\right|_{x=0}=0, \\
& \left.\left(-\lambda \nabla \mathcal{P}+\lambda_{l} \rho_{l} \mathbf{g}+\lambda_{g} \rho_{g} \mathbf{g}\right) \cdot \mathbf{n}\right|_{x=L}=V_{*}, \\
& \left.\mathcal{P}\right|_{t=0}=\mathcal{P}_{*}-\rho_{l} g x
\end{aligned}
$$

1. It results from Henry's law and $\mathcal{H} 3$ :

$$
d_{l} \mathcal{C} \equiv \partial_{t} \mathcal{C}+\mathbf{U}_{l} \nabla \mathcal{C}=h \partial_{t} \mathcal{P}+h \mathbf{U}_{l} \nabla \mathcal{P}=h \partial_{t} \mathcal{P}+\ldots
$$

2. The sum of equations $(1.1 a)$ and $(1.1 b)$ yields:

$$
\phi \partial_{t}\left(\rho_{l} \mathcal{S}+\rho_{g}(1-\mathcal{S})\right)+\nabla \cdot\left(\rho_{l} \mathbf{V}_{l}+\rho_{g} \mathbf{V}_{g}\right)=-\phi \rho_{g} q_{g}-\phi \rho_{l} q_{l}
$$

Differentiating by parts $(1.1 b)$ and $(*)$ we obtain:

$$
\begin{aligned}
& (1-\mathcal{C})\left(\phi \rho_{l} \partial_{t} \mathcal{S}+\rho_{l} \nabla \cdot \mathbf{V}_{l}+\phi \rho_{l} q_{l}\right)-\phi \rho_{l} \mathcal{S} d_{l} \mathcal{C}=0 \\
& \left(-\phi \rho_{g} \partial_{t} \mathcal{S}+\phi(1-\mathcal{S}) \partial_{t} \rho_{g}+\rho_{g} \nabla \cdot \mathbf{V}_{g}+\phi \rho_{g} q_{g}\right)+\left(\phi \rho_{l} \partial_{t} \mathcal{S}+\rho_{l} \nabla \cdot \mathbf{V}_{l}+\phi \rho_{l} q_{l}\right)+\ldots=0
\end{aligned}
$$

This leads to:

$$
\begin{aligned}
& \phi \partial_{t} \mathcal{S}+\nabla \cdot \mathbf{V}_{l}+\phi q_{l}=\frac{\phi \mathcal{S}}{(1-\mathcal{C})} d_{l} \mathcal{C}=\{(1.16)\}=\frac{\phi \mathcal{S} h}{(1-\mathcal{C})} \partial_{t} \mathcal{P} \equiv-\mathcal{M} \\
& -\phi \partial_{t} \mathcal{S}+\frac{\phi(1-\mathcal{S})}{\mathcal{P}} \partial_{t} \mathcal{P}+\nabla \cdot \mathbf{V}_{g}+\phi q_{g}=-\frac{\phi r \mathcal{S}}{(1-\mathcal{C})} d_{l} \mathcal{C}=r \mathcal{M}
\end{aligned}
$$

3. Summing (1.17a) and (1.17b) we obtain the second equation in (1.10).

4. Replacing $\mathbf{V}_{l}$ in $(1.17 a)$ by $\mathbf{V}$ through (1.4), we obtain:

$$
\phi \partial_{t} \mathcal{S}+\nabla\left(\mathcal{F} \mathbf{V}-\lambda_{g} \mathcal{F} \Delta \rho \mathbf{g}\right)=\frac{\phi \mathcal{S} h}{(1-\mathcal{C})} \partial_{t} \mathcal{P}-\phi q_{l}
$$


5. Multiplying the second equation $(1.10)$ by $\mathcal{F}$ and subtracting it from (**), we obtain the first equation (1.10).

6. Using the second equation in (1.10) and the relationship (1.6) between the velocity and pressure, we can remove the velocity and obtain the differential equation (1.15a) for pressure. Conditions $(1.15 b),(1.15 c)$ and $(1.15 d)$ result from (1.7).

\subsection{Derivation of the model. Step II: separation of pressure}

At the second step we separate completely the pressure, by applying hypotheses $\mathcal{H} \mathbf{1}$ and $\mathcal{H} \mathbf{2}$ and the asymptotic technique.

For the case of slow depletion: $\mathrm{t}_{h} \sim \mathrm{t}_{p} \ll \mathrm{t}_{*}$

Let us consider the equations (1.10) and (1.15) with conditions (1.7) in the scale of time $t \sim \mathrm{t}_{h}$. Then the terms with $q_{l}$ and $q_{g}$ will be of order $\varepsilon$, as well as the terms $\rho_{l} g$ and $\rho_{g} g$ in (1.15). Then, for saturation, pressure and total velocity we have the following asymptotic expansion:

$$
\mathcal{S}=1+\varepsilon \mathcal{S}_{1}+\ldots, \quad \mathcal{P}=\mathcal{P}_{*}+\varepsilon \mathcal{P}_{1}(x, t)+\ldots, \quad \mathbf{V}=\varepsilon \mathbf{V}_{1}+\ldots
$$

where $\mathcal{P}_{1}$ results from $(1.15)$ :

$$
\left\{\begin{array}{l}
\lambda_{l 0} \nabla \cdot\left(-\nabla \mathcal{P}_{1}+\rho_{l} \mathbf{g} / \varepsilon\right)=-\frac{\phi h\left(r_{*}-1\right)}{\left(1-\mathcal{C}_{*}\right)} \partial_{t} \mathcal{P}_{1}-\phi q / \varepsilon \\
\left.\lambda_{l 0}\left(-\nabla \mathcal{P}_{1}+\rho_{l} \mathbf{g} / \varepsilon\right) \cdot \mathbf{n}\right|_{x=0}=0,\left.\quad \lambda_{l 0}\left(-\nabla \mathcal{P}_{1}+\rho_{l} \mathbf{g} / \varepsilon\right) \cdot \mathbf{n}\right|_{x=L}=V_{*} / \varepsilon, \\
\left.\mathcal{P}_{1}\right|_{t=0}=-\rho_{l} g x / \varepsilon
\end{array}\right.
$$

where $\lambda_{l 0}=\left.\lambda_{l}(\mathcal{S})\right|_{\mathcal{S}=1}, \quad r_{*}=r\left(\mathcal{P}_{*}\right), \quad \mathcal{C}_{*}=\mathcal{C}\left(\mathcal{P}_{*}\right)$.

Returning to pressure $\mathcal{P}$ through $\mathcal{P}_{1}=\left(\mathcal{P}-\mathcal{P}_{*}\right) / \varepsilon$ and introducing the new variable $p=$ $\mathcal{P}-\mathcal{P}_{*}+\rho_{l} g x$, we obtain the problem (1.12).

Note that equations (1.10) may also be expand, but this is not necessary since the objective of pressure separation has been already reached.

For the case of fast depletion: $\mathrm{t}_{p} \ll \mathrm{t}_{h} \sim \mathrm{t}_{*}$

Let us consider (1.10), (1.15) and (1.7) in the scale of time $t \sim \mathrm{t}_{h} \sim \mathrm{t}_{*}$. Then all the terms in (1.15a) except the first one will be of order $\varepsilon$. Then we obtain immediately that the zero term of pressure is independent of $x: \mathcal{P}_{0}=\mathcal{P}(t)$. Then we have the following asymptotic expansion:

$$
\mathcal{S}=\mathcal{S}_{0}(x, t)+\varepsilon \mathcal{S}_{1}(x, t)+\ldots, \quad \mathcal{P}=\mathcal{P}_{0}(t)+\varepsilon \mathcal{P}_{1}(x, t)+\ldots, \quad \mathbf{V}=\varepsilon \mathbf{V}_{1}(x, t)+\varepsilon^{2} \ldots
$$

To obtain $\mathcal{P}_{0}$, it is better to use the integral relationship resulting from $(1.15 a)$ by integrating it over $x$ :

$$
-\left.\left(\lambda \partial_{x} \mathcal{P}+\lambda_{l} \rho_{l} g+\lambda_{g} \rho_{g} g\right)\right|_{x=0} ^{x=L}=-\int_{x=0}^{x=L}\left(\left(\frac{\mathcal{S C}(r-1)}{1-\mathcal{C}}+1-\mathcal{S}\right) \frac{\phi}{\mathcal{P}} \partial_{t} \mathcal{P}+\phi q\right) d x
$$

Substituting the asymptotic expansion and boundary-value conditions, we obtain:

$$
-V_{*}-\phi q L=\left(\frac{\overline{\mathcal{S}} \mathcal{C}\left(\mathcal{P}_{0}\right)\left(r\left(\mathcal{P}_{0}\right)-1\right)}{1-\mathcal{C}\left(\mathcal{P}_{0}\right)}+1-\overline{\mathcal{S}}\right) \frac{\phi L}{\mathcal{P}_{0}} d_{t} \mathcal{P}_{0}
$$

where $\overline{\mathcal{S}} \equiv \frac{1}{L} \int_{x=0}^{x=L} \mathcal{S}(x, t) d x$ 
Taking into account the closure relationships for the concentration and density, we obtain:

$$
\frac{d \mathcal{P}_{0}}{d t}\left(\frac{h \overline{\mathcal{S}}\left(\rho_{l}-\gamma \mathcal{P}_{0}\right)}{\gamma \mathcal{P}_{0}\left(1-h \mathcal{P}_{0}\right)}+\frac{1-\overline{\mathcal{S}}}{\mathcal{P}_{0}}\right)=-\left(\frac{V_{*}}{\phi L}+q\right)
$$

which is (1.13).

\subsection{Hyperbolicity of the model (1.10)}

Equations (1.10) may be presented as

$$
\left\{\begin{array}{l}
\phi \partial_{t} \mathcal{S}+V \partial_{x} \mathcal{F}-\partial_{x}\left(\lambda_{g} \mathcal{F} \Delta \rho g\right)=\mathcal{H} \\
\partial_{x} V=\mathcal{I}
\end{array}\right.
$$

where $\mathcal{M} \equiv \frac{\phi \mathcal{C} \mathcal{S}}{\mathfrak{T}(1-\mathcal{C})}, \quad \mathcal{H} \equiv-\mathcal{M}(1-\mathcal{F}+r \mathcal{F})-\frac{\phi \mathcal{F}(1-\mathcal{S})}{\mathfrak{T}}+\phi q_{g} \mathcal{F}-\phi q_{l}(1-\mathcal{F}), \quad \mathcal{I} \equiv$ $(r-1) \mathcal{M}+\frac{\phi(1-\mathcal{S})}{\mathfrak{T}}-\phi q$.

This system of two equations of first order with respect to $\mathcal{S}$ and $V$ can be represented in the form of Cauchy-Kowalevski, explicit for the derivatives $\partial_{x}$ :

$$
\partial_{x} \mathbf{U}+\mathbf{A} \otimes \partial_{t} \mathbf{U}=\mathbf{B}
$$

where

$$
\mathbf{U}=\left(\begin{array}{c}
\mathcal{S} \\
V
\end{array}\right), \quad \mathbf{A}=\left(\begin{array}{cc}
\alpha \phi & 0 \\
0 & 0
\end{array}\right), \quad \mathbf{B}=\left(\begin{array}{c}
\alpha \mathcal{H} \\
\mathcal{I}
\end{array}\right), \quad \alpha \equiv \frac{1}{V \mathcal{F}^{\prime}-\Delta \rho g\left(\lambda_{g} \mathcal{F}\right)^{\prime}}
$$

where the symbol "prime" stands for the derivative with respect to $\mathcal{S}$.

If the denominator in $\alpha$ is nonzero for all $\mathcal{S}$, then the eigenvalues of matrix $\mathbf{A}$ exist and are real: $\xi_{1}=0$ and $\xi_{2}=\alpha \phi$, and there exists an orthonormal basis of the left eigenvectors of matrix A: $l^{1}=(1,0)$ and $l^{2}=(0,1)$. Then system (1.19) is hyperbolic.

\section{Gas-liquid segregation in a closed reservoir}

Using the model (1.10), we will analyze the impact of the phase transitions on the process of gas-liquid segregation. First of all, we will analyze the impact of the source terms only. At the second step, the impact of the non-constant total velocity will be studied (section 3 ).

\subsection{Problem setup}

Consider a closed reservoir, such that $V_{g *}=V_{l *}=0$. The production of the fluid occurs throughout the overall thickness and is determined by the rates $q_{l}$ and $q_{g}$. The main feature of such a system consists of the fact that the total velocity $\mathbf{V}$ is zero, which will be shown below.

Let the liquid and gas extraction rates be proportionate, i.e., the production rate of each phase is proportional to its fractional flow: $\frac{q_{l}}{q_{g}}=\frac{(1-\mathcal{F})}{\mathcal{F}}$. Then the term $\mathrm{V}$ in (1.10) disappears. In practice, this means that gas and liquid are extracted by the same producing well, therefore their rates depend on each other. For pressure, we will use the asymptotic solution (1.12).

The problem of segregation resulting from (1.10) and (1.12) is reduced to a single equation for 

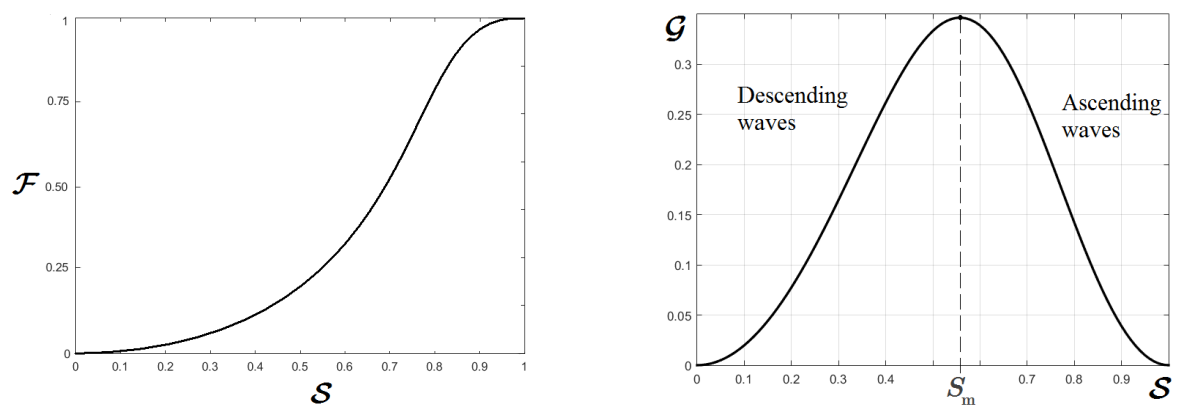

FIGURE 1. Diagrams $\mathcal{F}(\mathcal{S})$ and $\mathcal{G}(\mathcal{S})$ for the problem of gas-liquid segregation

the saturation:

$$
\begin{aligned}
& \left\{\begin{array}{l}
\partial_{t} \mathcal{S}-\partial_{x} \mathcal{G}(\mathcal{S})=-\mathcal{H}(\mathcal{S}), \quad 0<x<L, \quad 0<t<\mathrm{t}_{*} \\
\left.\mathcal{S}\right|_{t=0}=1, \quad \forall x \\
\left.\mathcal{G}(\mathcal{S})\right|_{x=0}=0,\left.\quad \mathcal{G}(\mathcal{S})\right|_{x=L}=0, \quad \forall t
\end{array}\right. \\
& \left\{\begin{aligned}
\mathcal{G}(\mathcal{S}) & =\lambda_{g}(\mathcal{S}) \mathcal{F}(\mathcal{S}) \Delta \rho g \phi^{-1} \\
\mathcal{H}(\mathcal{S}) & =\frac{\mathcal{S C}(1-\mathcal{F}+r \mathcal{F})}{\mathfrak{T}(1-\mathcal{C})}+\frac{\mathcal{F}(1-\mathcal{S})}{\mathfrak{T}}
\end{aligned}\right.
\end{aligned}
$$

where $\Delta \rho, r$ and $\mathcal{C}$ depend on pressure, which is a parameter of the problem defined through (1.12), which has, in this case, the explicit analytical solution independent of $x: p=p(t)=$ $-\frac{\left(1-\mathcal{C}_{*}\right)}{h\left(r_{*}-1\right)} \int_{0}^{t} q d t^{\prime}$, so that:

$$
V \equiv 0, \quad \mathcal{P}=\mathcal{P}_{*}-\rho_{l} g x-\frac{\left(1-\mathcal{C}_{*}\right)}{h\left(r_{*}-1\right)} \int_{0}^{t} q d t^{\prime}
$$

Parameter $\mathfrak{T}$ is: $\mathfrak{T}=-\left(\frac{1}{\mathcal{P}} \partial_{t} \mathcal{P}\right)^{-1}=\frac{\mathcal{C}\left(r_{*}-1\right)}{q\left(1-\mathcal{C}_{*}\right)}$.

Function $\mathcal{G}(\mathcal{S})$ describes the gas transport due to the buoyancy force, while the right-hand side $(-\mathcal{H})$ describes the decrease in liquid saturation in the overall domain due to liquid degassing and gas expansion. Function $\mathcal{F}(\mathcal{S})$ is the fractional flow of liquid without gravity.

Functions $\mathcal{F}(\mathcal{S})$ and $\mathcal{G}(\mathcal{S})$ are shown in Fig. 1. Note that their derivatives are zero at points $\mathcal{S}=0$ and $\mathcal{S}=1$, according to the properties of the relative permeabilities. The derivative $d \mathcal{G} / d \mathcal{S}$ determines the speed of the wave propagation and its direction. One distinguishes two families of waves:

- propagating upwards $\left(\mathcal{G}^{\prime}<0\right)$, which corresponds to high liquid saturation. Physically, this is caused by gas rise due to the buoyancy force.

- propagating downwards $\left(\mathcal{G}^{\prime}>0\right)$, which corresponds to high gas saturation. Such a wave is the result of gas expansion.

\subsection{Steady-state solution}

Equation (2.1) has steady-state solutions, which satisfy the ordinary differential equation:

$$
\partial_{x} \mathcal{G}(\mathcal{S})=\mathcal{H}(\mathcal{S}), \quad 0<x<L,
$$




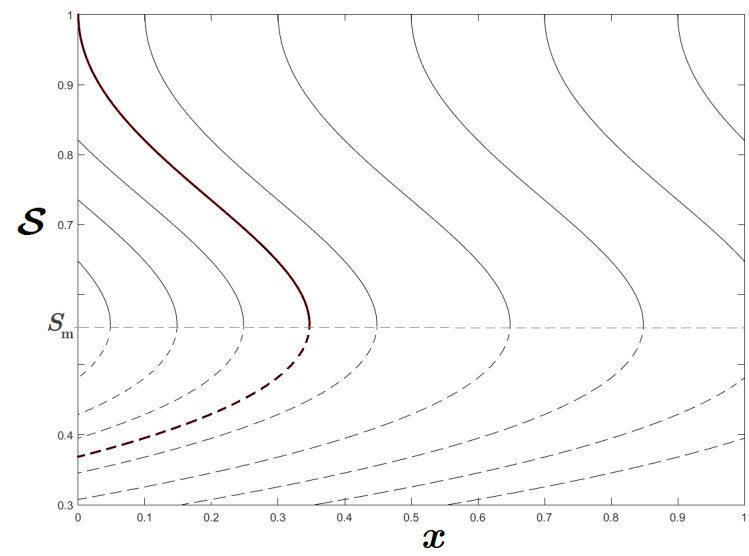

FIGURE 2. Steady-state solutions of the problem of gas-liquid segregation

and which are the result of the equilibrium between the wave propagation (term $\partial_{x} \mathcal{G}$ ) and the gas generation (term $\mathcal{H})$.

Its solutions are shown in Fig. 2.

These solutions are non single-valued and have a singularity at point $\mathcal{S}=\mathcal{S}_{m}$ of the maximum of the function $\mathcal{G}(\mathcal{S})$, in which the derivative $d \mathcal{S} / d x$ becomes infinite. Only the upper part of these curves above the dashed line has a physical meaning. It represents the equilibrium between gas rise, which leads to the increase of the local liquid saturation, and the gas generation, which leads to the decrease of $\mathcal{S}$.

The red curve, which corresponds to the boundary condition $\left.\mathcal{S}\right|_{x=0}=1$, represents the main interest. We will show that this curve makes part of the non stationary solutions.

\subsection{General structure of the solution of (2.1)}

The boundary condition in (2.1) means that the saturation at point $x=L$ is either $\mathcal{S}=1$, or $\mathcal{S}=0$. The case $\mathcal{S}=1$ has no meaning (no gas at the reservoir top), consequently it is expected that $\left.\mathcal{S}\right|_{x=L}=0, \forall t>0$. In other words, the free gas appears immediately at the reservoir top, by creating the initial shock of saturation between $\mathcal{S}=1$ on the left of it and $\mathcal{S}=0$ on the right. Therefore, one expects to have discontinuous solutions.

Consequently, the solution of the boundary-initial problem for quasi-linear hyperbolic equation (2.1) is expected to consist of four elements:

(i) a rarefaction/compression wave ("rc-wave"), which is a nontrivial continuous function presented by a fragment of the curve $\mathcal{G}(\mathcal{S})$ and propagating at the velocity $\mathcal{G}^{\prime}(\mathcal{S})$ along the characteristics $x=x(t)$ defined by the system of equations:

$$
\frac{d \mathcal{S}}{d t}=-\mathcal{H}(\mathcal{S}), \quad \frac{d x}{d t}=-\mathcal{G}^{\prime}(\mathcal{S})
$$

which are different for various $\mathcal{S}$;

(ii) a stationary rc-wave determined by the problem (2.3), which is the equilibrium between gas rise and gas generation. It does not move but lengthens in time.

(iii) a plateau, which is constant in $x$ but varies in time. It satisfies the problem:

$$
\partial_{t} \mathcal{S}=-\mathcal{H}(\mathcal{S}),\left.\quad \mathcal{S}\right|_{t=0}=1
$$

For a fixed time moment it is represented by a point in the diagram $\mathcal{G}(S)$.

(iv) a shock, which is a strong discontinuity between two rc-waves, or between a rc-wave and a plateau, or between two plateaux. It is determined by the following conditions: 


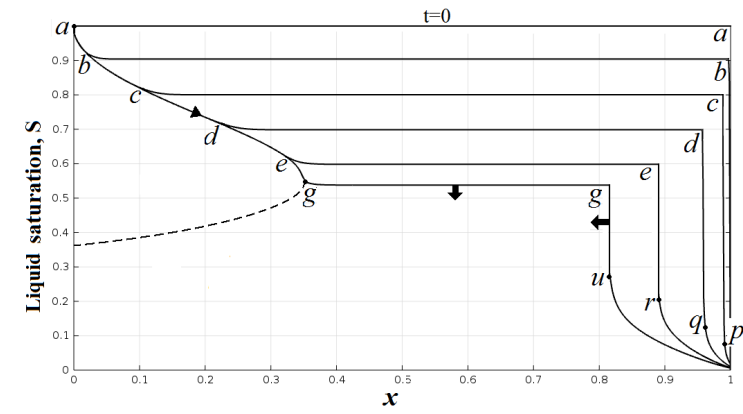

(a)

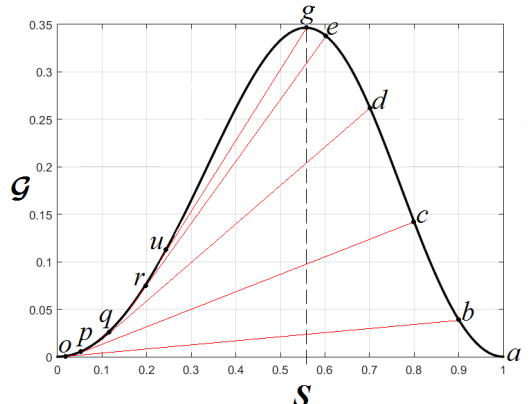

(b)

FIGURE 3. Variation of the liquid saturation at the first stage (a) and the corresponding diagram $\mathcal{G}(\mathcal{S})$ (b). The dashed curve is the stationary wave

- the mass-balance equation (the Hugoniot-Rankine condition) relating the shock velocity $U_{s}$, the saturation behind it $\mathcal{S}^{-}$and ahead of it $\mathcal{S}^{+}$:

$$
U_{s}=\frac{\mathcal{G}^{+}-\mathcal{G}^{-}}{\mathcal{S}^{+}-\mathcal{S}^{-}}
$$

In the diagram $\mathcal{G}(\mathcal{S})$ the shock velocity is determined by the tangent of the straight line relating the points behind the shock $\left(\mathcal{G}^{-}, \mathcal{S}^{-}\right)$and ahead of it $\left(\mathcal{G}^{+}, \mathcal{S}^{+}\right)$.

- the entropy conditions of Lax and Oleinik, which requires the shock velocity to be between the velocities of the rc-waves that are connected to it and to not cross the curve $\mathcal{G}(\mathcal{S})$. If the shock is connected to only one mobile rarefaction wave, the entropy condition implies that the shock velocity must be equivalent to the rc-wave velocity at the contact point. Graphically this means that the straight line $(2.5)$ is tangent to the curve $\mathcal{G}(\mathcal{S})$ at point of contact with a mobile rc-wave.

\subsection{Solution at the first stage}

First of all, we consider the case when the coefficients of (2.1) do not depend on pressure, i.e., $\mathcal{P}=\mathcal{P}_{*}$, then $\Delta \rho, r$ and $\mathcal{C}$ are constant.

The solution of the problem is obtained analytically, by applying the method of characteristics. Knowing all the structural elements of the solution mentioned in section 2.3 , we can calculate their velocities and saturations at the points of junction by using analytical or semi-analytical relationships obtained previously: (2.3), (2.4), (2.5). The ordinary differential equations have been solved by using Mathlab.

The solution of the problem is presented in Fig. 3.

The average value of the derivative $\mathcal{G}^{\prime}(\mathcal{S})$ of the convective term in (2.1) tends to zero (according to Fig. 1), then the "average" equation (2.1) has the form (2.4), whose solution is a plateau variable in time. This corresponds to the saturation decrease in the reservoir due to progressive liquid degassing. Consequently, the main element of the solution becomes a set of plateaux $a a, b b, c c, d d, e e, g g$, At the same time, the buoyancy force, governed by the convective term, maintains a high liquid saturation at the reservoir bottom $(\mathcal{S} \rightarrow 1$, at $x \rightarrow 0)$ and low liquid saturation at the reservoir top $(\mathcal{S} \rightarrow 0$, at $x \rightarrow L)$.

At the reservoir top, gas is accumulated, i.e., $\mathcal{S}=0$, as mentioned in section 2.3. The connection between the low saturation and the plateaux occurs through a shock, whose progressive positions in space are $b o, c p, d q, e r, g u$. This shock represents the wave reflected from the reservoir top and propagating downwards. It appears, since the rc-waves connecting a high and a low saturation produce non single-valued solutions. Indeed, the rc-wave $c-d-e-g-u-r-$ $q-p-o-0$, which passes from point $c$ of the plateau $c c$ to point $(\mathcal{G}, \mathcal{S})=(0,0)$, has negative 


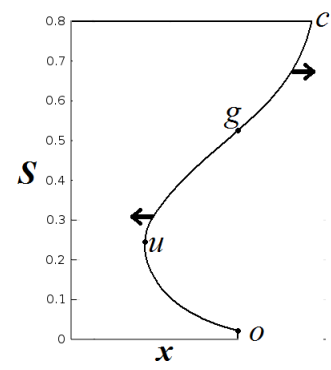

FIGURE 4. Example of a non single-valued solution described by the rc-wave $c-d-e-g-u-\ldots-o$

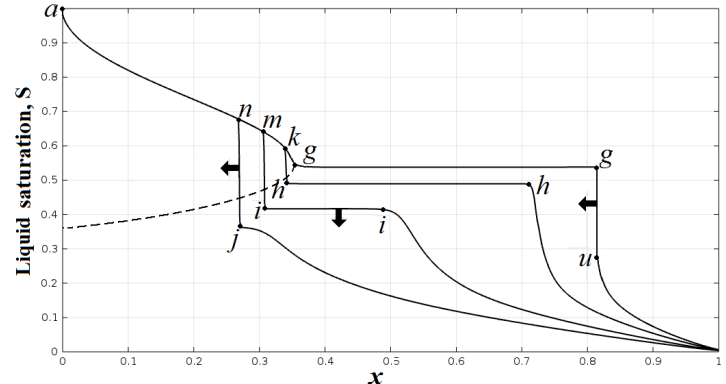

(a)

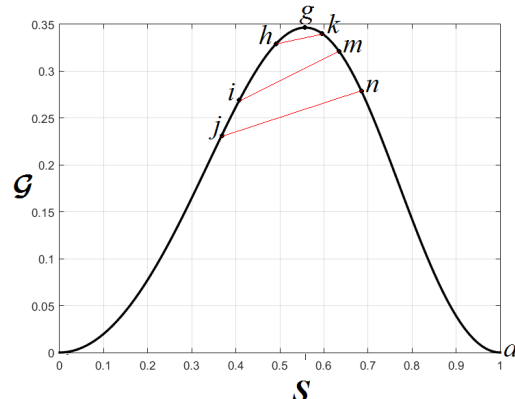

(b)

FIGURE 5. Variation of the liquid saturation at the second stage (a) and the corresponding diagram $\mathcal{G}(\mathcal{S})$

(b)

transport velocity for high saturations (points $c, d, e$ ), but positive velocity for low saturations (points $u, r, q, p, o$ ), which implies the following formal solution in terms of $\mathcal{S}(x)$ (Fig. 4). Such a solution is not single-valued, can not exist and must be replaced by a shock.

Shock $b o-c p-d q-e r-g u$ marks the boundary of the gas cap. It has positive velocity, i.e., is transported downwards, which means the growth of the gas cap. The shock velocity increases in time. At points of connection with the rc-wave $(o, p, q, r, u$ the straight line of this shock is tangent to the curve $\mathcal{G}(\mathcal{S})$, according to the entropy conditions.

The total system of equations that defines these shocks is: Eq. (2.5), in which $\mathcal{S}^{+}$is the solution of (2.4), while $\mathcal{S}^{-}$is the solution of $\mathcal{G}^{\prime}\left(\mathcal{S}^{-}\right)=U_{s}$, which is the entropy condition. This system of equations is closed and determines the shock in a unique way.

At the reservoir bottom, gas saturation is low, the process is controlled by the right-hand part of the diagram $\mathcal{G}(\mathcal{S})$, which corresponds to the gas rise. The connection between the high saturation and the plateaux occurs via a stationary rc-wave described by (2.3) at the boundary condition $\left.\mathcal{S}\right|_{x=0}=1$. It passes through points $a, b, c, d, e, g$ along the right-hand branch of the curve $\mathcal{G}(\mathcal{S})$.

The first stage continue up to the moment when the solution reaches the singular point $g$, at which $\mathcal{S}=\mathcal{S}_{m}$, and which corresponds to the final point of the steady-state rc-wave.

\subsection{Solution at the second and the third stages}

At the reservoir bottom, after the stationary rc-wave $a-b-c-d-e-g$ reaches the singular point $g$ (at which $S=\mathcal{S}_{m}$ ), the gas expansion becomes more important than the gas rise, which leads to the appearance of a descending wave. This waves is however non single-valued, similar to preceding paragraph, and transforms into a new shock propagating downwards (Fig. 5). Its consecutive positions are shown as $h k, i m$, and $j n$. This shock connects the plateau and the stationary wave, which enables us to determine its parameters in a unique way by (2.5), in which 
$\mathcal{S}^{+}$is the solution of (2.3) with the boundary condition $\left.\mathcal{S}\right|_{x=0}=1$, while $\mathcal{S}^{-}$is determined by (2.4).

At the reservoir top, the gas cap boundary becomes less sharp but more continuous, which means the transformation of the shock $g u$ into a rc-wave propagating downwards. The transport velocity of this wave is determined by the tangents lines to the left-hand part of the function $\mathcal{G}(\mathcal{S})$, which are, obviously, larger than the velocities of the shock $h k$-im-jn. Consequently, the upper rarefaction waves should collide with the down shock.

After the collision, the plateau disappears. The solution has a single shock, which continue to propagate downward. This is the third stage. Curve $1-j-n-a$ marks the beginning of this stage.

\subsection{Comparison with numerical solution}

The comparison of the analytical solutions presented above has been made with numerical solutions obtained by two different methods. One of them is based on finite-element discretisation of the domain, which is realized in simulator Comsol Multiphysics, while the second one is based on the finite volumes implemented in the open source code DuMux. Both methods use the fully implicit scheme of discretisation in time (the fully implicit means that all coefficients and the right-hand part are implicit), which is unconditionally stable.

Comsol Multiphysics allows the use on non-sturctured grids, but the discrete schemes are non necessary conservatives, as for any finite-element method.

DuMux (Flemisch et al. (2011)) is based on the algebraic code DUNE (Distributed and Unified Numerics Environment) Bastian et al. (2008) and the module MpNc (M-phases, N-components) that simulates the multicomponent and multiphase flow and transport in porous media. The spatial discretization is done by the Box-method, which is the finite volume method (vertexcentered) based on non-structured non-orthogonal grids. The principle ideas of this method were already presented in Patankar (1980) in terms of the "control volume techniques". The more modern version may be found in Kramer (Nicholas) and Bank (Rose). The finite volume method traditionally uses orthogonal grids, since in this case the expressions for fluxes through the faces of finite elements are greatly simplified. The use of non-orthogonal grids (as in the method of finite elements) is a significant advantage of the Box-method.

For one-dimensional problem studied herein, both methods lead to close numerical schemes.

For hyperbolic systems having discontinuous solutions, both methods apply the technique of low diffusion.

Two cases are presented in Fig. 6 and 7.

The characteristic times have been selected as follows: $t_{h}=0.5, t_{*}=2$. The dimensionless parameters that determine the process are: $\varepsilon=0.2, \rho^{0} g L / \mathcal{P}_{*}=0.2, \rho_{l} / \rho_{g}^{0}=5, \mathcal{C}_{l}^{0}=0.2$, $\gamma / \mathcal{P}_{*}=0.2, h / \mathcal{P}_{*}=0.2, \phi=0.2$. The perturbation time is $t_{p}=0.4$ when $\varepsilon=0.2$, or $\mathrm{t}_{p}=0.8$ when $\varepsilon=0.4$, and so on.

Despite the satisfactory results, we should note that the total velocity $V$ is not exactly constant, as predicted by the asymptotic model. The Fig. 8 shows the behaviour of the dimensionless total velocity for four moments of time.

Note that the maximal/minimal values of these fluctuations are very small, between -0.001 and 0.003 .

\subsection{Structure of the gas cap}

According to the results obtained, the formation of a gas cap passes through three stages shown in Fig. 9. 


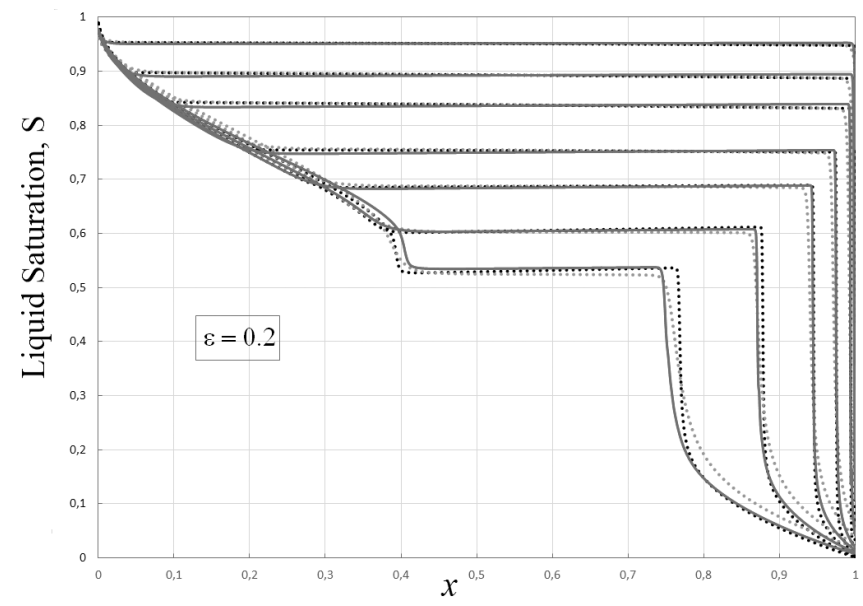

FIGURE 6. Comparison of the analytical solution (the solid lines) with numerical simulations by DuMux (the black points) and Comsol Multiphysics (the grey points), for $\varepsilon=0.2$

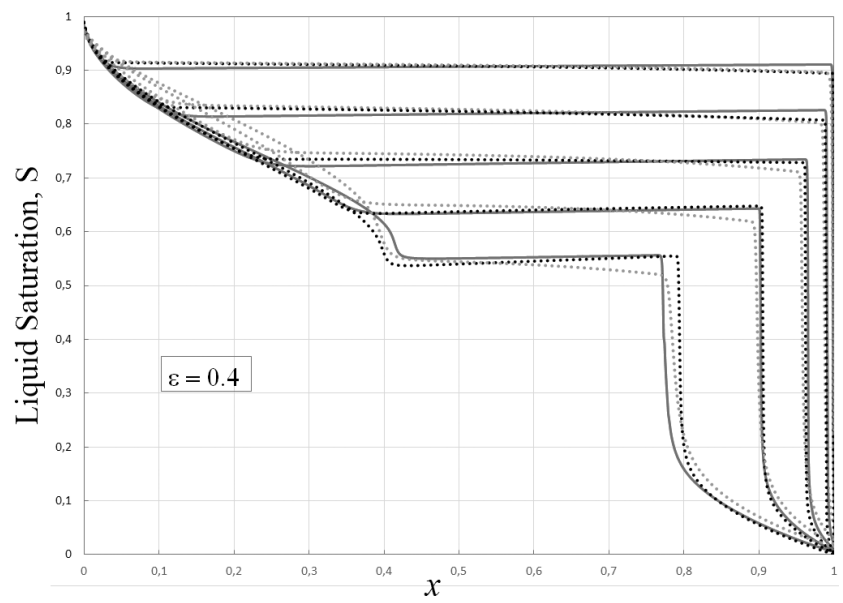

FIGURE 7. Comparison of the analytical solution (the solid lines) with numerical simulations by DuMux (the black points) and Comsol Multiphysics (the grey points), for $\varepsilon=0.4$

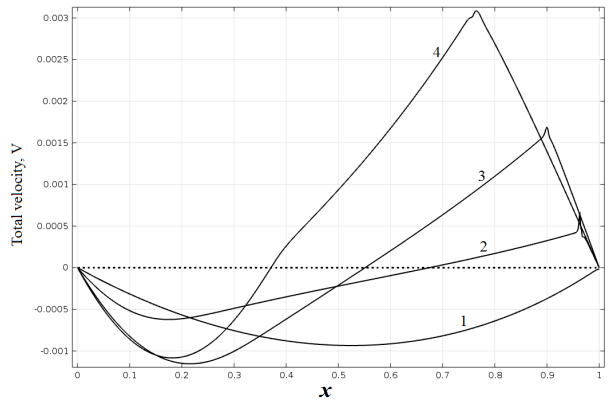

FIGURE 8. Total velocity, exact numerical results for four moments of time 

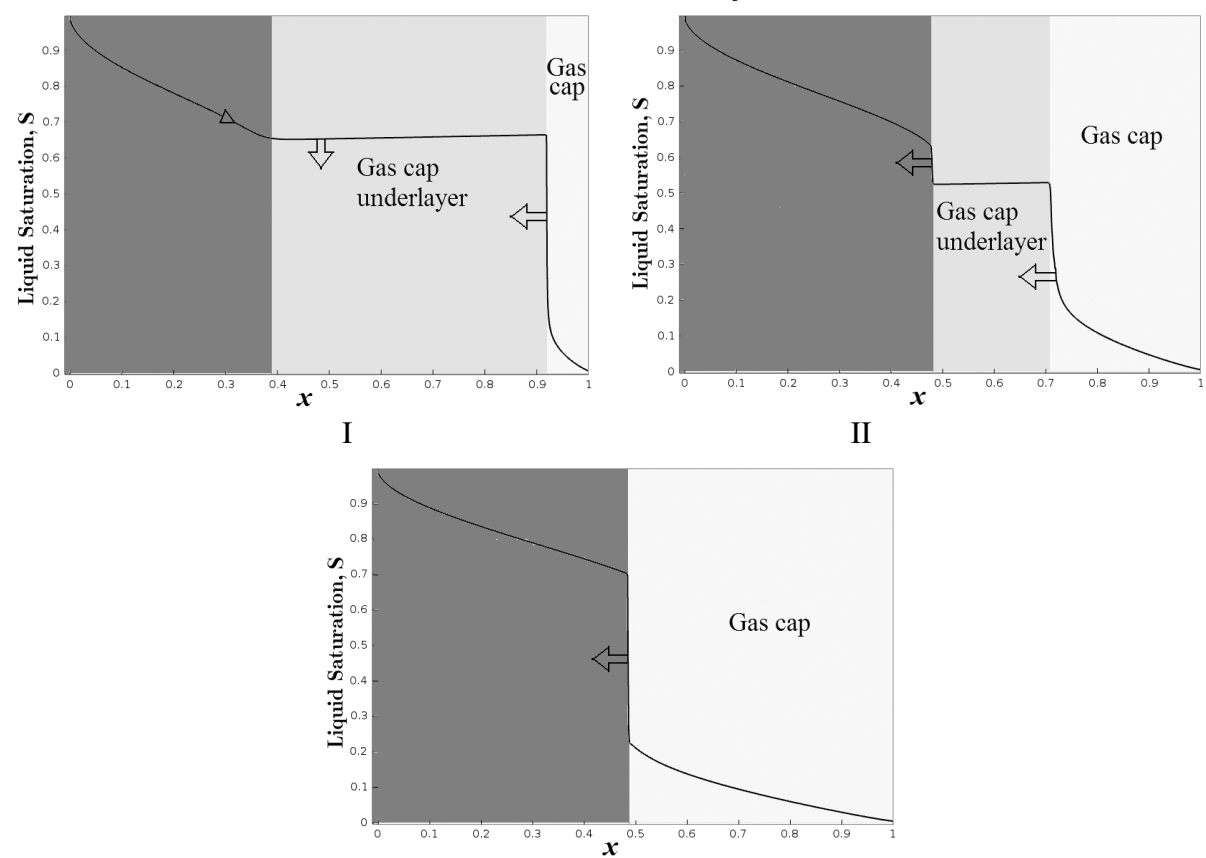

III

FIGURE 9. Evolution of the gas cap structure in time: stages I, II and III

As seen, the zone at the bottom, highly saturated with liquid, is separated from the gas cap by a gas cap underlayer, in which the gas saturation is almost invariable in space. The growth of the gas cap leads to the contraction of this underlayer, while keeping almost constant the thickness of the liquid zone.

\section{Impact of the non-zero total velocity}

\subsection{Problem formulation}

Let the fluid be extracted just from the reservoir top. Then in the problem (1.12): $\left.V\right|_{x=L}=$ $V *$, and $q_{l}=q_{g}=0$. Let, as previously, the liquid and gas extraction rates be proportionate, i.e. $\frac{V_{l *}}{V_{g *}}=\frac{(1-\mathcal{F})}{\mathcal{F}}$.

Then the problem of segregation resulting from (1.10) and (1.12) becomes:

$$
\left\{\begin{array}{l}
\partial_{t} \mathcal{S}+\frac{V}{\phi} \partial_{x} \mathcal{F}-\partial_{x} \mathcal{G}(\mathcal{S})=-\frac{\mathcal{C S}(1-\mathcal{F}+r \mathcal{F})}{(1-\mathcal{C}) \mathfrak{T}}-\frac{\mathcal{F}(1-\mathcal{S})}{\mathfrak{T}}, \\
\partial_{x} V=\frac{\phi \mathcal{C} \mathcal{S}(r-1)}{(1-\mathcal{C}) \mathfrak{T}}+\frac{\phi(1-\mathcal{S})}{\mathfrak{T}}, \\
\left.\mathcal{S}\right|_{t=0}=1,\left.\quad \mathcal{G}(\mathcal{S})\right|_{x=0}=0,\left.\quad \mathcal{G}(\mathcal{S})\right|_{x=L}=0,\left.\quad V\right|_{x=0}=0
\end{array}\right.
$$

where $\mathcal{G}(\mathcal{S})=\frac{1}{\phi}\left(\lambda_{g} \mathcal{F} \Delta \rho g\right)$, and parameter $\mathfrak{T}(x, t)$ is $\mathfrak{T}^{-1}=-\frac{1}{\mathcal{P}} \partial_{t} \mathcal{P} \sim-\frac{1}{\mathcal{P}_{*}} \partial_{t} \mathcal{P}$.

In this system the pressure is determined from the independent problem (1.12) formulated for 
function $p=\mathcal{P}-\mathcal{P}_{*}+\rho_{l} g x$ :

$$
\left\{\begin{array}{l}
\lambda_{l 0} \Delta p=\omega \partial_{t} p, \quad 0<x<L, \quad t>0 \\
\left.p\right|_{t=0}=0,\left.\quad \partial_{x} p\right|_{x=0}=0,\left.\quad \partial_{x} p\right|_{x=L}=-\frac{V_{*}}{\lambda_{l 0}}
\end{array}\right.
$$

which has an approximate analytical solution obtained by the integral method of KarmanPohlhausen:

$$
\begin{aligned}
& p=\left\{\begin{array}{l}
\frac{V_{*}}{2 \lambda_{l 0}}\left[\frac{(x-L)^{2}}{l-L}-2(x-L)+\varphi(t)-L\right], \quad l(t) \leqslant x \leqslant L, \\
0, \quad 0 \leqslant x<l(t)
\end{array}\right. \\
& l(t)=\left\{\begin{array}{l}
L-\sqrt{\frac{6 \lambda_{l 0}}{\omega V_{*}} \int_{0}^{t} V_{*}\left(t^{\prime}\right) d t^{\prime}}, \quad 0 \leqslant t \leqslant \mathrm{t}_{p} \\
0, \quad t>\mathrm{t}_{p}
\end{array}\right. \\
& \varphi(t)=\left\{\begin{array}{l}
l(t), \quad 0 \leqslant t \leqslant \mathrm{t}_{p} \\
\frac{L}{3}-\frac{V_{*}\left(\mathrm{t}_{p}\right) L}{3 V_{*}}-\frac{2 \lambda_{l 0}}{\omega L V_{*}} \int_{\mathrm{t}_{p}}^{t} V_{*}\left(t^{\prime}\right) d t^{\prime}, \quad t>\mathrm{t}_{p}
\end{array},\right.
\end{aligned}
$$

where $t_{p}$ is determined as the solution to the equation:

$$
\frac{1}{V_{*}} \int_{0}^{\mathrm{t}_{p}} V_{*}(t) d t=\frac{L^{2} \omega}{6 \lambda_{l 0}}
$$

Calculating the derivative $\partial_{t} p$ from (3.3) we obtain the explicit relationship for $\mathfrak{T}$ :

$\mathfrak{T}^{-1}=\left\{\begin{array}{l}\frac{V_{*}}{2 \lambda_{l 0} \mathcal{P}_{*}}\left[\left(\frac{x-L}{l-L}\right)^{2} \frac{d l}{d t}-\frac{d \varphi}{d t}\right]-\frac{1}{2 \lambda_{l 0} \mathcal{P}_{*}}\left[\frac{(x-L)^{2}}{l-L}-2 x+\varphi+L\right] \frac{d V_{*}}{d t}, \quad l \leqslant x \leqslant L \\ 0, \quad 0 \leqslant x<l\end{array}\right.$

In the case $V_{*}=$ const, we obtain $\mathrm{t}_{p}=\frac{L^{2} \omega}{6 \lambda_{l 0}}$, while the functions $l(t)$ and $\mathfrak{T}(x, t)$ become:

$$
\begin{aligned}
& l(t)=\left(L-\sqrt{\frac{6 \lambda_{l 0} t}{\omega}}\right) \mathcal{H}\left(\mathrm{t}_{p}-t\right), \\
& \mathfrak{T}^{-1}=\left\{\begin{array}{l}
\frac{V_{*}}{4 \mathcal{P}_{*}} \sqrt{\frac{6 \lambda_{l 0}}{\omega t}}\left[1-\left(\frac{x-L}{l-L}\right)^{2}\right] \mathcal{H}\left(\mathrm{t}_{p}-t\right)+\frac{V_{*}}{\mathcal{P}_{*} \omega L} \mathcal{H}\left(t-\mathrm{t}_{p}\right), \quad l \leqslant x \leqslant L \\
0, \quad 0 \leqslant x<l
\end{array}\right.
\end{aligned}
$$

where $\mathcal{H}(t)$ is the Heaviside function.

\subsection{Derivation of the solution to the problem for pressure}

The solution of (3.2) may be obtained by the method of integral relationships of KarmanPohlhausen. 


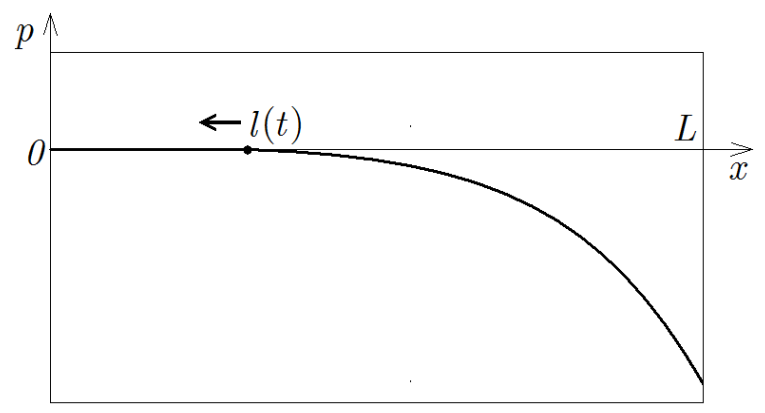

FIGURE 10. Approximation of the function $p(x, t)$, defined through (1.13), in the method of Karman-Pohlhausen

Let us assume that the pressure is perturbed in the finite zone $l(t)<x \leqslant L$ (the perturbation comes from point $x=L$ ), as shown in Fig. 10, where $l(t)$ is the unknown mobile coordinate.

Within this zone, the pressure is a polynomial function which satisfies the boundary-value conditions and the differential equation (1.13) in the average:

$$
\begin{aligned}
& p=\left\{\begin{array}{l}
-\frac{V_{*}}{\lambda_{l 0}}\left[a(t)(x-L)^{2}+b(t)(x-L)+c(t)\right], \quad l(t) \leqslant x<L, \\
0, \quad 0 \leqslant x<l(t)
\end{array}\right. \\
& \left.p\right|_{x=l}=0,\left.\quad \partial_{x} p\right|_{x=l}=0,\left.\quad \partial_{x} p\right|_{x=L}=-\frac{V_{*}}{\lambda_{l 0}},\left.\quad p\right|_{t=0}=0, \\
& \left.\lambda_{l 0} \partial_{x} p\right|_{x=l} ^{x=L}=\omega \partial_{t} \int_{l}^{L} p d x
\end{aligned}
$$

Then we obtain for functions $a(t), b(t), c(t)$ and $l(t)$ :

or

$$
\begin{gathered}
a(l-L)^{2}+b(l-L)+c=0, \quad 2 a(l-L)+b=0, \quad b=1, \\
-V_{*}=\omega \partial_{t}\left[\frac{V_{*}}{\lambda_{l 0}}\left(\frac{a(l-L)^{3}}{3}+\frac{(l-L)^{2}}{2}+c(l-L)\right)\right]
\end{gathered}
$$

$$
a=-\frac{1}{2(l-L)}, \quad c=-\frac{(l-L)}{2}, \quad-6 V_{*} \lambda_{l 0}=\omega \partial_{t}\left(V_{*}(l-L)^{2}\right)
$$

The last equation can be integrated, which gives: $(l-L)^{2}=-\frac{6 \lambda_{l 0}}{\omega V_{*}} \int_{0}^{t} V_{*}\left(t^{\prime}\right) d t^{\prime}$. This leads to $(3.3)$.

\subsection{Results of solution}

The solution of the problem is presented in Fig. 11.

As seen, the upper shock appears, as in the previous example, but remains immobile during all the period. The gas cap is determined by the lower shock, which progressively moves down.

\subsection{Conclusions}

In the present paper we have developed the wave model for liquid saturation and the total velocity for gas-liquid flow with phase transitions in porous media, which is an extension of the Buckley-Leverett model. The impact of the phase transitions is manifested in the appearance of 


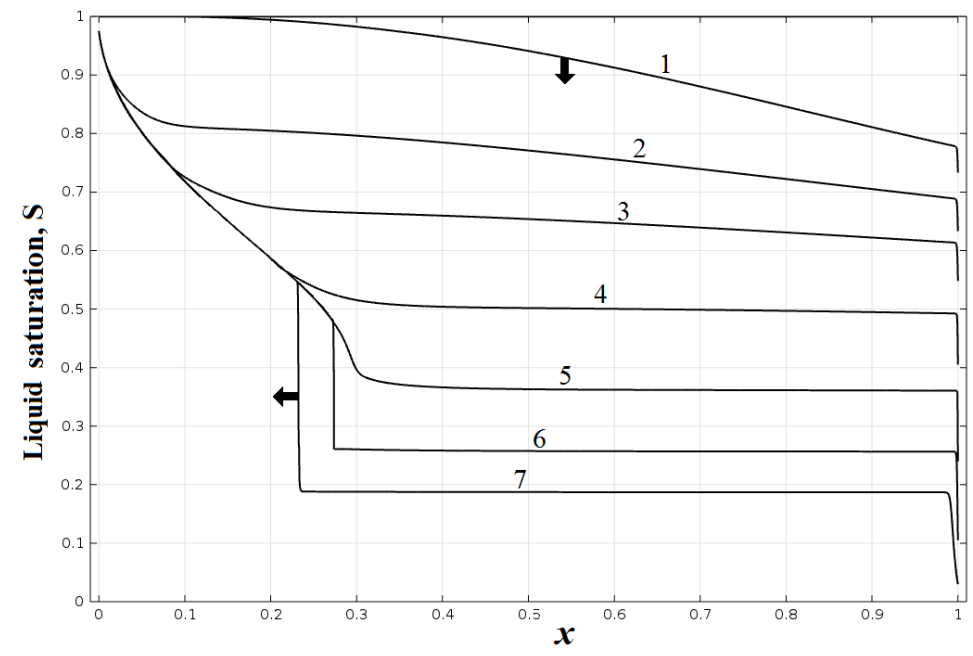

FIGURE 11. Evolution of the liquid saturation in the case of gas fluid production from the reservoir top, for seven moments of time

the source terms and in the non-constant total velocity. In the general case, the model consists of two equations, which have been shown to be hyperbolic.

In contrast to the traditional approach when the reduction of the equations of multicomponent two-phase flow to a wave model is based on the assumption of ideal mixing within each phase (the phase volume is equal to the sum of the individual volumes of pure components) and the assumption of invariable or linear pressure, we obtained the hyperbolic system (1.10) for variable pressure, and without the assumption of ideal mixing. The separation of pressure is done in two asymptotic cases (fast and slow gas ascension) and the property of a very fast propagation of pressure perturbation.

This model was used to analyse the impact of phase transitions to gas-liquid segregation. The fluid was assumed to consists of two chemical components. We solved two different problems, for the first time just to analyse the impact of the source terms, and the other problem had the objective to analyze the impact of the non-zero total velocity. In both cases we solved analytically the independent problem for pressure.

We have shown the complex structure of the solutions of these two problems consisted of several fragments: rarefaction waves, a steady-state wave, plateaux and shocks. Due to the obtained model the interfaces between the phases can be treated in terms of the shock waves governed by Hugoniot-Rankine conditions and entropy conditions.

The exact numerical simulations have been performed by applying two different methods: finite elements and finite volumes. The comparison has proved the satisfactory behaviour of the asymptotic solutions even for sufficiently large values of $\varepsilon$.

The importance of the obtained reduced model is in the fact that it provides exact analytical solutions and detects exactly several strong discontinuities in the structure of the solution, which is impossible to do if we solve numerically the unreduced model. Consequently, the analytical solutions obtained can be used as a reference to find the best numerical schemes for solving the non-reduced equations.

\section{REFERENCES}

Bank R., Rose D., and Fichtner W. Numerical methods for semiconductor device simulation, IEEE Transactions on Electron Devices, 30, 9 (1983), 1031-1041.

Bastian P., Blatt M., Dedner A., Engwer C., Klofkorn R., Kornhuber R., Ohlberger M., Sander O. A generic 
grid interface for parallel and adaptive scientific computing. part II: Implementation and tests in DUNE. Computing, 82(23), (2008) 121138.

Bedrikovetsky P. Mathematical theory of oil and gas recovery, Kluwer Academic Publishers, 1993.

Buckley S.E., Leverett M.C., et al.. Mechanism of fluid displacement in sands. Trans. AIME, 146 (1942), 107-116.

Chraïbi M. Modlisation de l'expasion de gaz dissout dans les huiles lourdes en milieux poreux. Ph.D. thesis, Université Pirerre et Marie Curie, 31 janvier, 2008 (thesis director S. Zaleski).

Entov V.M. Nonlinear waves in physicochemical hydrodynamics of enhanced oil recovery. Multicomponent flows. In: Porous media : physics, models, simulation: proceedings of the international conference. Moscow, Russia, 19-21 November 1997, page 33. World Scientific Pub Co Inc, 2000.

Entov V.M., and Zazovsky A. Hydrodynamics of enhabced oil recovery. Ed. Nedra, Moscow, 1989 (in Russian).

Flemisch B., Darcis M., Erbertseder K., Faigle B., Lauser A., Mosthaf K., Muthing S., Nuske P., Tatomir A., Wolff M. et al. DuMux: DUNE for multi-phase, component, scale, physics,... flow and transport in porous media. Adv Water Resour, 34(9), (2011) 11021112.

Kalisch H., Mitrovic D., and Nordbotten J.M. Non-standard shocks in the Buckley-Leverett equation. $J$. Math. Anal. Appl., 428 (2015), 882-895.

Kramer K., Nicholas G., and Hitchon W. Semiconductor Devices, a Simulation Approach. Prentice Hall Professional Technical Reference, 1997.

F.M. Orr. Theory of gas injection processes. Ed. Stanford University, California, 2002.

Panfilov M. Quasi-equilibrium asymptotics of the depletion of subsurface petroleum and gas strata. Doklady Academii Nauk SSSR, 31(5), (1986), 225-227.

Panfilov M., Zaleski S., and Josserand C. Mechanisms of formation of natural hydrogen reservoirs in thermal aquifers: impact of methanogen bacteria and the non equilibrium of gas bubble dynamics. Procs. ECMOR-15: $15^{\text {th }}$ European Conference on Mathematics of Oil Recovery, Amsterdam, the Netherlands, August 29 - Septembre 1, 2016, paper WE GRA 06.

Patankar S.V. Numerical Heat Transfer and FLuid FLow. McGraw-Hill Book Company, Washington - New York - London, 1980.

H. Rhee, R. Aris, and N.R. Amundson, First-order partial differential equations, Volume 1, Printice-Hall, 1986.

Young R. Two-phase geothermal flows with conduction and the connection with Buckley-Leverett theory. Transport in Porous Media, 12(3) (1993), 261-278. 